R. J. Cohen and W. T. Sullivan, III, eds.

\title{
Light Pollution: Education of Students, Teachers and the Public
}

\author{
John R. Percy \\ Erindale Campus, University of Toronto \\ Mississauga ON Canada L5L 1 C6
}

\begin{abstract}
The preservation of the astronomical environment is intimately connected to society's understanding and appreciation of astronomy. This requires effective education of students, teachers and the general public. We know how this can be done. It remains for us to become education-active and to convince our colleagues and students to do likewise.
\end{abstract}

\section{Introduction}

Many speakers at this Symposium have pointed out that education is the first step in preserving the astronomical sky. In fact, education is essential for the health of all of astronomy, both to attract and train the next generation of astronomers and to promote awareness, understanding and appreciation of astronomy on the part of the taxpayers who support us. The IAU has sponsored over 375 Symposia and Colloquia; only two of these have dealt with education!

The process of astronomical education is made more complicated (and interesting) by the fact that education takes place in a variety of situations - not just in the classroom (Fraknoi 1996). Furthermore, there are vast differences in the systems of education in different countries and even within different countries. IAU Commission 46 (Teaching of Astronomy), with its system of National Representatives, may be able to help. But astronomy education should not be left to educators alone: if every astronomer spent an additional 1 per cent of their time on education and public outreach, the effect could be profound.

Light pollution is obviously undesirable because it is a symptom of inefficient, ineffective lighting; major cities waste millions of dollars each year in lighting the night sky. This hinders the work of professional and amateur astronomers and robs everyone of dark skies. Is this a problem? Only if astronomy, and dark skies, are in some way "useful".

\section{Why Is Astronomy Useful?}

Astronomy is deeply rooted in history and culture, as a result of its practical applications and its religious and philosophical implications. Among the scientific revolutions of history, astronomy stands out. Recent lists of "the hundred most important people of the millenium" invariably include a few astronomers. Astronomy still governs the cycle of day and night, the seasons, and many as- 
pects of long-term climate change; impacts of asteroids and comets have been implicated in mass biological extinctions. It contributes to the advancement of mathematics and computer science, and science and technology. It is a dynamic science in its own right; each year, many of the most important scientific discoveries are in astronomy and related fields. Astronomy deals with our place in time and space, and with our cosmic roots. It promotes environmental consciousness, both through images of our fragile planet taken from space, and through the knowledge that we may be alone in the universe. It also has aesthetic and emotional dimensions: it reveals a universe which is vast and beautiful; it has inspired artists and poets for centuries; it harnesses curiosity, imagination, and a sense of shared exploration and discovery. In school, it can be used to teach concepts such as light and gravitation, to give students a more meaningful appreciation of scales of distance and time, and to illustrate the observational approach to the scientific method. It is the ultimate interdisciplinary subject, and "crosscurricular connections" are highly-valued in modern curriculum development. It attracts young people to science and technology; it increases public awareness and interest in science; and it is an enjoyable hobby for millions of people worldwide. In the words of Henri Poincaré: "Astronomy is useful because it shows how small our bodies, how large our minds".

\section{Astronomy Education - Where and How Does It Occur?}

Education can be divided into: (1) formal or school education, and (2) informal or public education. In fact, these two overlap: the public derive their basic knowledge of (and attitudes toward) astronomy in the schools, and students learn as much about astronomy out of school as within. As Fraknoi (1996) has eloquently pointed out, astronomy education "happens in hundreds of planetaria and museums around the country; it happens at meetings of amateur astronomy groups; it happens when someone reads a newspaper or in front of television and radio sets; it happens while someone is engrossed in a popular book on astronomy, or leafs through a magazine like $S k y \mathscr{E}$ Telescope; it happens in youth groups taking an overnight hike and learning about the stars; and it happens when someone surfs the astronomy resources on the internet. When we consider astronomy education, its triumphs and tribulations, we must be sure that we don't focus too narrowly on academia, and omit the many places that it can and does happen outside the classroom".

It follows that education about light pollution will be most effective when it comes through a coalition or partnership - the local "astronomical community". This includes professional astronomers; scientists, engineers, and other academics in related fields; students at every level; educators at every level, in every setting; journalists; amateur astronomers; environmentalists; and interested members of the general public. This provides a "critical mass" of concerned citizens, providing a broad base of support.

\section{Light Pollution and Formal Education}

First - a brief introduction to formal science and astronomy education. We know many things about effective science teaching and learning: young people (and 
adults) have deep-seated misconceptions about scientific topics, which teachers must identify and deal with; scientific concepts must be introduced in a logical sequence, and at an age when students can absorb them; and science teaching is most effective when an inquiry-based or activity-based approach is taken (see various papers in Percy 1996). This suggests that sky-watching ("eyes-on astronomy") should be an important part of astronomy education. Even though day-time astronomy is more convenient than night-time astronomy ("the stars come out at night, the students don't"), observing the night sky should be part of every astronomy course. This can be achieved, for instance, by giving each student a star map, or by allowing every student to make their own planisphere from a template. They can be taught how to use this in the classroom, so that they can use it in the evening at their convenience.

Unfortunately, there are many barriers to the effective teaching of astronomy: lack of appreciation of its value; lack of understanding of astronomy and astronomy education by teachers; and the ingrained "classical" methods of teaching by memorization and regurgitation. This is true at all levels, including university; effective professional development for teachers is essential.

We should all work to get more and better astronomy in the school curriculum. In many countries, astronomy is part of the school science curriculum; it is part of the US National Science Education Standards (NRC 1996) and the Canadian equivalent. Simple observable changes (such as day and night) are covered in the lower grades of elementary school, followed by a more detailed discussion of solar system topics (such as moon phases) in the upper grades. In the lower grades of secondary school, the curriculum may include material on the planets, sun, stars and galaxies. In the upper grades, astronomy may be used to illustrate physics topics such as gravity, light and spectra.

Ironically, the study of light pollution makes a very good "cloudy night activity" for students. It can be done in an urban or suburban setting. It does not require clear skies. It helps students to understand scientific, technological and societal issues. See Percy (1998a) for a review of some educational activities and projects on light pollution. Light pollution may not be an explicit "topic" in the science curriculum, but the science curriculum includes processes, skills and applications, which can be taught effectively through the study of light pollution and transferred to other science topics.

Because light pollution is such a promising topic for promoting science education, and because Metaxa (this Symposium) was directing a successful light pollution project in the schools in Greece, Crawford, Metaxa and Percy (1998) recently produced a special teachers' newsletter on the topic - an issue of the Astronomical Society of the Pacific's The Universe in your Classroom. This newsletter is sent to thousands of classrooms across the world; it is available on the internet (www.aspsky.org/html/tnl/44/lightpoll.html); and it is translated into a dozen languages. In addition to background information and resource lists on light pollution, it includes three kinds of activities:

- Astronomy activities in which students investigate the effect of light pollution and other factors on the limiting magnitude of stars which they can see. 
- Physics activities in which students use simple transmission diffraction gratings to observe the spectra of various natural and artificial light sources in their local environment. Sets of gratings for an entire class can be obtained at low cost from various science supply companies.

- Science-and-society activities in which students investigate the types of lighting in their community, the effectiveness of the lighting, and the channels which they can use to promote better lighting.

In my own province of Ontario, Canada, the school science curriculum has recently been revised, and astronomy and space are now a part of the curriculum in grade 6 (age 11 years) and grade 9 (age 14 years). One of the new textbooks (Plumb et al. 1999) includes a two-page section by Alan Hirsch on the issue of light pollution. Several hundred thousand students will be exposed to this material over the life of the textbook. If the astronomical community could provide appropriate information, activities and resources on light pollution to textbook authors and publishers, there would be a greater chance that this material would be presented to students. To illustrate the impact of textbook authors: Professor Jay Pasachoff, an astronomer at Williams College USA, not only writes textbooks for the 200,000 post-secondary students who take introductory astronomy courses in North America each year, but also co-authors textbooks for high school, junior high school and elementary school; he also writes astronomy guidebooks for the general public. This is a powerful contribution to astronomy education, which should not go unnoticed or unused. Many other professional astronomers also author excellent textbooks for these purposes.

Perhaps the best way to generate effective educational material would be through a formal science education project, tied to documents such as the US National Science Education Standards (NRC 1996) and carried out with input and assistance from educators. In the US, the National Science Foundation funds a variety of science education projects and programmes. I urge organizations like the International Dark-Sky Association, and astronomers throughout the world, to find and use such funding to develop the best possible education material. Astronomers and school-teachers in France have been especially successful in developing effective educational materials.

Other threats to the astronomical environment - pollution of the electromagnetic spectrum, and space debris - also fit naturally in the school science curriculum. The electromagnetic spectrum is introduced in almost every secondary school and university astronomy course, but there is seldom any specific discussion of which radio wavelengths are used for astronomy and which for everyday practical and commercial applications. The specific contributions of radio astronomy should be highlighted. Likewise, almost every astronomy textbook discusses orbital motion in the earth's gravitational field and shows a diagram of near-earth space. A discussion of space debris - where it is and how it moves - would provide a natural application.

\section{Light Pollution and Informal Education}

Since other speakers at this Symposium have discussed public education about light pollution, I will mention a few specific topics only. 
The Mass Media. The mass media have a profound effect on public understanding of, and attitudes to, science. Scientists often complain about media coverage (or lack thereof) of science topics. The situation can be helped if scientists learn why and how the media operate, and work with them to improve science coverage. In my experience, media coverage of light pollution has been accurate and sympathetic - perhaps because it is an issue to which everyone can relate. During a recent (July 1999) joint meeting of the Astronomical Society of the Pacific, the Royal Astronomical Society of Canada and the American Association of Variable Star Observers, in Toronto, there were three major articles about Ontario's new "dark sky reserve", including a front-page article in the Globe \& Mail, which styles itself "Canada's national newspaper". (The Torrance Barrens Conservation Reserve is a provincial park; dark skies will be one of its aims and one of its attractions.)

Specialized Media. As well as the "mass media", there are scientific magazines which have a very large readership and influence - National Geographic and Scientific American, for instance. Preservation of the astronomical environment would be an interesting and appropriate topic for either of these publications and would have tremendous impact; priority should be given to getting articles into such publications.

Amateur Astronomers. There are various definitions of amateur astronomer. The simplest is "someone who enjoys astronomy and cultivates it as a hobby". They may be content to read about astronomy, attend lectures, or watch TV documentaries. They may enjoy sky-gazing and be adept in using star charts and telescopes. Williams (1988) has proposed a more stringent definition: "someone who does astronomy with a high degree of skill, but not for pay". Such individuals can make important contributions to astronomical research, through the measurement of variable stars, for instance, or the discovery of comets, asteroids, novae and supernovae. They can also make important contributions to astronomical education (Percy 1998b): they produce radio and TV programmes; they write newspaper and magazine articles and books; they give astronomy courses and lectures; they volunteer in planetaria, science centres and public observatories; they organize star parties (an excellent means of educating the public about light pollution); they are the driving force behind International Astronomy Day. They can even contribute to astronomy education in the schools, through programmes such as the Astronomical Society of the Pacific's Project ASTRO (Bennett et al. 1998). The number of "master" amateur astronomers in North America is comparable with the number of professional astronomers; the total number of astronomy hobbyists is $10-50$ times greater (Gada et al. 1999). They are certainly our "grass-roots allies" in the battle against light pollution; see Percy (1998a) for specific examples.

Other Means of Informal Education. Planetaria, of course, are the last bastions of darks skies in the cities. They are especially appreciated by young people. When Toronto's McLaughlin Planetarium closed, several years ago, the most eloquent lament was written by a student, in the University newspaper. Astronomy programmes in parks, camps, youth groups (Boy Scouts and Girl Scouts, for instance) and outdoor education centres are also an ideal way of introducing people to the beauty of dark skies. We should remember, though, that not everyone has the opportunity to travel to these dark-sky locations. 
Education should begin with astronomers themselves: every astronomy library and ideally every astronomer's bookshelf, should include a complete set of IDA Factsheets and reprints of key articles on light pollution.

Educating the world about astronomy and about light pollution is a major challenge which can be met if we work together with like-minded organizations. In all parts of the world, this concept of "partnership" can lead to "astronomy education initiatives" which can provide both a rationale and a possible source of funding for education about the preservation of the astronomical environment. IAU Commission 50 (for the professional astronomy community) and the International Dark-Sky Association (for the "astronomical community" in general) can play a key role in these partnerships; we should all support their work.

Acknowledgements. My participation in IAU Symposium 196 was made possible by a research grant from the Natural Sciences and Engineering Research Council of Canada. I thank David Crawford, Margarita Metaxa and Syuzo Isobe for many interesting and useful discussions.

\section{References}

Bennett, M., Fraknoi, A., \& Richter, J. 1998, in New Trends in Teaching Astronomy, ed. L. Gouguenheim et al., Cambridge University Press, 249

Crawford, D., Metaxa, M., \& Percy, J.R. 1998, "Light Pollution", special issue of The Universe in your Classroom, ASP, San Francisco CA

Fraknoi, A., 1996, in Astronomy Education: Current Developments, Future Coordination, ed. J.R. Percy, ASP Conf. Series. 89, 9

Gada, A., Stern, A., \& Williams, T.R. 1999, paper presented at AmateurProfessional Partnership in Astronomical Research and Education, Toronto, Canada, 5 July 1999

National Research Council 1996, "National Science Education Standards", National Academy Press, Washington DC

Percy, J.R., (ed.) 1996, Astronomy Education: Current Developments, Future Coordination, ASP Conf. Series. 89

Percy, J.R. 1998a, in Preserving the Astronomical Windows, ed. S. Isobe \& T. Hirayama, ASP Conf. Series 139,7

Percy, J.R., 1998b, in New Trends in Teaching Astronomy, ed. L. Gouguenheim et al., Cambridge University Press, 205

Plumb, D., Ritter, B., James, E., \& Hirsch, A. 1999, Science 9, ITP Nelson, Toronto, Canada

Williams, T.R., 1988, in Stargazers: The Contribution of Amateurs to Astronomy, ed. S. Dunlop \& M. Gerbaldi, Springer-Verlag, 24 